invadens under anaerobic and aerobic conditions. Antimicrob Agents Chemother. 1976;9:696-700.

6 Muller M, Meingassner JG, Miller WA, Ledger WJ. Three metronidazole-resistant strains of Trichomonas vaginalis from the United States. Am $₹$ Obstet Gynecol 1980;138:808-12.

7 Smith RF, DiDomenico A. Measuring the in vitro susceptibility of Trichomonas vaginalis to metronidazole. Sex trasm Dis 1980;7:120-4.

Accepted for publication 18 December 1992.

\section{STD Diagnostics Initiative}

Sexually transmitted diseases (STDs) are an important cause of morbidity and mortality worldwide. In many developing countries the incidence of treatable STDs continues to rise and in some countries has reached epidemic proportions. Both genital ulcer disease and non-ulcerative STDs are associated with a three- to five-fold increased risk of human immunodeficiency virus (HIV) transmission.

In light of the prevalence of STDs in many countries, work is ongoing to develop or strengthen STD control programs in many parts of the world. An integral part of this effort is the identification, development, and introduction of affordable diagnostic tests appropriate for use in resource-limited health care settings, particularly those serving women of reproductive age in whom most infections are asymptomatic. To adequately meet global need, these diagnostics must be:

- Inexpensive - cost to the provider of less than US $\$ 1.00$ per patient

- Simple-minimal training and simple or no equipment required

- Rapid-results available before patient leaves clinic

- Convenient-specimens simple to collect, socioculturally acceptable, minimal preparation required

- Stable-reagents have long shelf life, no refrigeration required

- Functional-packaged simply, low cost

- Accurate-appropriately sensitive and specific, taking into account the potential morbidity and cost associated with undetected infection and the cost of treatment

To stimulate and sustain the development and/or adaptation of appropriate STD diagnostics, the STD Diagnostics Initiative was formed in November 1990. The goal of this multilateral Initiative is to facilitate the development and distribution of affordable diagnostic tests appropriate for use in resource-limited settings and to help integrate these tests into STD prevention and control programs. In working toward this goal, the Initiative interacts with researchers, manufacturers, STD and maternal and child health program managers, clinicians, laboratory workers, and representatives of international health and donor agencies. At present, the Initiative's interests are focussed on:

- Chlamydial and gonococcal diagnostic tests or indicators, primarily for women, which would reduce the incidence of reproductive tract complications of infection and the expense of treatment.
Simple diagnostic tests or simplified test procedures for STD pathogens (i.e. Treponema pallidum, Haemophilus ducreyi, HSV) which are associated with increased risk of HIV transmission.

- Simple indicators which can be effectively used with syndromic algorithms.

Serological tests for $T$ pallidum, HSV-2, and $H$ ducreyi for epidemiological surveillance and evaluation in STD/AIDS control programs.

For more information about the Initiative, please contact the Secretariat for the STD Diagnostics Initiative, Program for Appropriate Technology in Health (PATH), 4 . Nickerson Street, Seattle, Washington 98109-1699, U.S.A.

SETH BERKLEY Chairman, Steering Committee, STD Diagnostics Initiative

Accepted for publication 4 February 1993.

\section{HIV disease in Africans of high social class}

The majority of research on AIDS in Africa has been performed in Government institutions, which tend to be used by the poorest members of society. Few studies have elicited the clinical presentations of $\mathrm{HV}$ disease in those who are affluent, or have described the epidemiological features in this group. ${ }^{12}$

Discharge data were collected retrospectively on all admissions who were HIV positive on a general medical ward at the Aga Khan Hospital (AKH), Nairobi, Kenya, between 1 September 1990 and 28 February 1991. HIV testing was performed on clinical grounds. Epidemiological data were obtained by KER interviewing 23 consecutive HIV positive patients attending an outpatient clinic, using a structured questionnaire.

There were 1041 medical admissions (666 men, 375 women) and 121 (12\%) cases (116 patients) were HIV positive ( $15 \%$ of men, $5 \%$ of women). The number of negative HIV results is unknown. The admissions comprised 98 men (mean age 36.5 years, range 22-52) and 18 women (mean age 30.4 years, range 17-55). HIV status was previously unknown in $74 \%$. The presenting diagnoses are shown in the table. Neuropsychiatric

Table 1 Medical diagnoses in HIV positive patients

\begin{tabular}{llll}
\hline $\begin{array}{l}\text { Diagnosis } \\
\text { (\%) }\end{array}$ & $\begin{array}{l}\text { Male } \\
n=98\end{array}$ & $\begin{array}{l}\text { Female } \\
n=18\end{array}$ & $\begin{array}{l}\text { Total } \\
n=116\end{array}$ \\
\hline Pulmonary tuberculosis & 21 & 7 & $28(24)$ \\
Neuro-psychiatric disease & 14 & 2 & $16(14)$ \\
Herpes zoster & 7 & 2 & $9(8)$ \\
Gastroenteritis & 8 & 0 & $8(7)$ \\
Bacterial pneumonia & 8 & 1 & $9(8)$ \\
Malaria & 3 & 2 & $5(4)$ \\
Urinary tract infection & 5 & 0 & $5(4)$ \\
Pyrexia of unknown origin & 3 & 1 & $4(3)$ \\
Anaemia of unknown cause & 1 & 2 & $3(3)$ \\
Nephrotic syndrome & 1 & 1 & $2(2)$ \\
Salmonella typhi & 1 & 0 & $1(1)$ \\
Salmonella paratyphi & 1 & 0 & $1(1)$ \\
Histoplasmosis & 1 & 0 & $1(1)$ \\
Bubonic plague & 1 & 0 & $1(1)$ \\
Eczema & 1 & 0 & $1(1)$ \\
Perianal abscess & 1 & 0 & $1(1)$ \\
Oesophagitis & 1 & 0 & $1(1)$ \\
Hodgkin's disease & 1 & 0 & $1(1)$ \\
Unknown & 22 & 0 & $22(19)$ \\
\hline
\end{tabular}

